\title{
Systematic Study of a SiPMT array readout for fast time-of-flight detectors
}

\author{
M. Bonesini ${ }^{* 1}$;R. Bertoni ${ }^{1}$, O. Barnaba ${ }^{2}$, A. de Bari ${ }^{2}$, R. Nardó ${ }^{2}$, M. C. Prata $^{2}$, \\ M. Rossella ${ }^{2}$ \\ ${ }^{1}$ Sezione INFN Milano Bicocca, Dipartimento di Fisica G. Occhialini, Piazza Scienza 3, Milano, \\ Italy \\ ${ }^{2}$ Sezione INFN Pavia and Dipartimento di Fisica, Universitá di Pavia, via A. Bassi 6, Pavia, Italy
}

\begin{abstract}
Time-of-flight detectors, based on scintillation counters, may use conventional photomultipliers for the readout. Problems arise in presence of external magnetic fields. SiPMT arrays are insensitive to magnetic fields and may be a suitable option to replace photomultipliers. Timing performances for several types of SiPMT arrays have been studied and results are presented.
\end{abstract}

Technology and Instrumentation in Particle Physics 2014

2-6 June, 2014

Amsterdam, the Netherlands

\footnotetext{
* Speaker.

${ }^{\dagger}$ E-mail: maurizio.bonesini@mib.infn.it
} 


\section{Introduction}

Time-of-flight (TOF) detectors may be constructed with scintillator slabs arranged in a suitable geometry. In a scintillator counter, photodetector signals may be measured at both ends, for redundancy, and then pulse-height analyzed. Arrival times are transformed into standard logic levels with a discriminator module (constant fraction or leading edge) and then used as a STOP for a TDC.

As readout devices, photomultipliers (PMTs) are commomly used. They may have low jitter, fast rise time but are usually sensitive to external magnetic fields. Examples of recent fast time-offlight counters with photomultiplier readout are the one of the MICE experiment at RAL [1] where conventional Hamamatsu R4998 PMTs were used [2], the timing counter of the MEG experiment at PSI [3], [4] with fine-mesh Hamamatsu R5924 PMTs [5] and the big TOFW wall of the Harp experiment at CERN PS [6] with Phillips XP2020 PMTs.

A simple parametrization of the timing resolution for a TOF detector is given by :

$$
\sigma_{t}=\sqrt{\frac{\sigma_{s c i}^{2}+\sigma_{p h}^{2}+\sigma_{p l}^{2}}{N_{p e}}+\sigma_{\text {elec }}^{2}}
$$

where: $\sigma_{s c i}$ accounts for the scintillator response, $\sigma_{p h}$ for the photodetector jitter, $\sigma_{p l}$ for the path lengths variations and $\sigma_{e l e c}$ for the jitter of the electronic readout system. $N_{p e}$ is the average number of photoelectrons. The dominating factors for $\sigma_{t}$ are $N_{p e}$ and the counter dimensions (mainly its length L). For resolutions below $100 \mathrm{ps}$, contributions such as $\sigma_{p h}$ become increasingly important.

In this paper, the question if the readout with conventional Hamamatsu R4998 photomultipliers may be replaced by Silicon PMTs, using the MICE TOF system as a benchmark ${ }^{1}$ has been addressed.

Tests were done in laboratory with Bicron BC404 counters of dimension $600 \times 60 \mathrm{~mm}^{2}, 1$ " thick, equivalent to the ones of the TOF2 detector of MICE [7].

\section{SiPMT Array readout}

The SiPMT arrays under test are $4 \times 4$ arrays made of $3 \times 3 \mathrm{~mm}^{2}$ individual SiPMT from SenSL, Advansid and Hamamatsu and their main characteristics are shown in table 1.

Table 1: Main characteristics of used SiPMT arrays.

\begin{tabular}{|l|c|c|c|c|}
\hline & $V_{b r k}(V)$ & $\begin{array}{c}\text { Bias range } \\
\text { (over } V_{b r k)}\end{array}$ & $\lambda_{\max }(n m)$ & $\begin{array}{c}\text { PDE } \\
\text { (at } \lambda_{\max } \text { ) }\end{array}$ \\
\hline Hamamatsu S11828-3344 & $65 \pm 10$ & $1-4$ & 440 & $\sim 35 \%$ \\
SenSLArray SM-4-3035-CER & $27.5 \pm 0.5$ & $1-5$ & 500 & $\sim 20 \%$ \\
SenSLArray SB-4-3035-CER & $24.5 \pm 0.5$ & $1-5$ & 420 & $\sim 41 \%$ \\
Advansid ASD-SiPM3S-4x4A & $35 \pm 7$ & $2-7$ & 480 & $\sim 22 \%$ \\
\hline
\end{tabular}

The emission peak of the used Bicron BC-404 scintillator is around $408 \mathrm{~nm}$, best suited to the Hamamatsu or SenSL SB SiPMT's PDE, as we will see later.

\footnotetext{
${ }^{1}$ making no attempt to optimize the light-guides or other detector aspects
} 

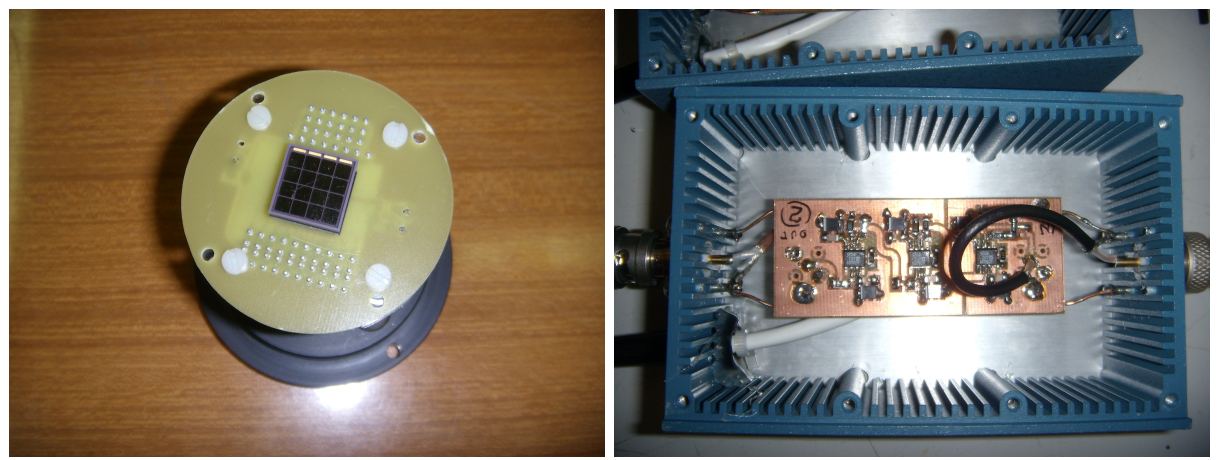

Figure 1: Left panel: image of one home-made PCB base. Right panel: image of one home-made amplifier.

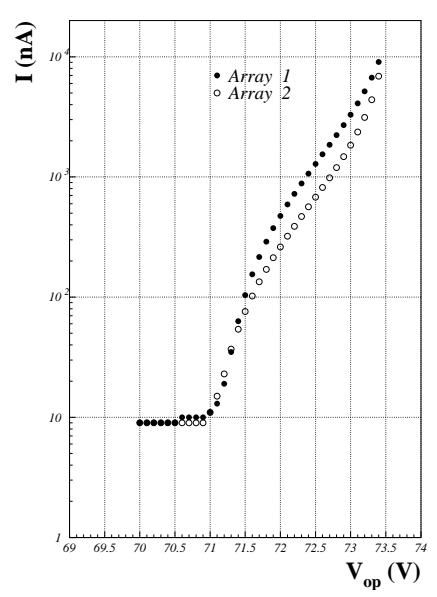

Figure 2: I-V curves for the Hamamatsu S11828-3344 array

The individual SiPMT of the array are connected in parallel, giving a single analogue summed output, on a home-made PCB base, shown in figure $1^{2}$. The output is then fed into a custom made inverting amplifier (also shown in figure 1), with pole zero suppression. The input dynamic range is between 0 and $70 \mathrm{mV}$ and the amplification factor is in the range 20-35 X (according to the level of pole zero compensation). The SiPMT arrays breakdown voltage was computed by measuring their I-V curves, using a Keithley 2600 sourcemeter ${ }^{3}$. An example of one I-V curve is shown in figure 2. The timing characteristics of the SiPMT arrays used for the readout of the scintillation BC404 test counter (signal risetime/falltime) have been studied with a wideband scope. Figure 3 shows, as an example, the shape of the signals from Hamamatsu (bottom) or SenSL (top) arrays, with a trigger on cosmic rays at the center of the counter. As SenSL arrays have larger values for risetimes and falltimes (at least a factor 2x), we concentrated our efforts on Hamamatsu and Advansid arrays.

\section{Experimental results}

Figure 4 shows the setup used for the tests. The light emitted from a Nichia NDHV310APC

\footnotetext{
${ }^{2}$ this simple choice increases the risetime of the output signal, as the single SiPMT capacities are summed in the time constant of the circuit

${ }^{3}$ with $0.02 \%$ accuracy on the set voltages and $0.03 \%$ accuracy on the measured currents
} 


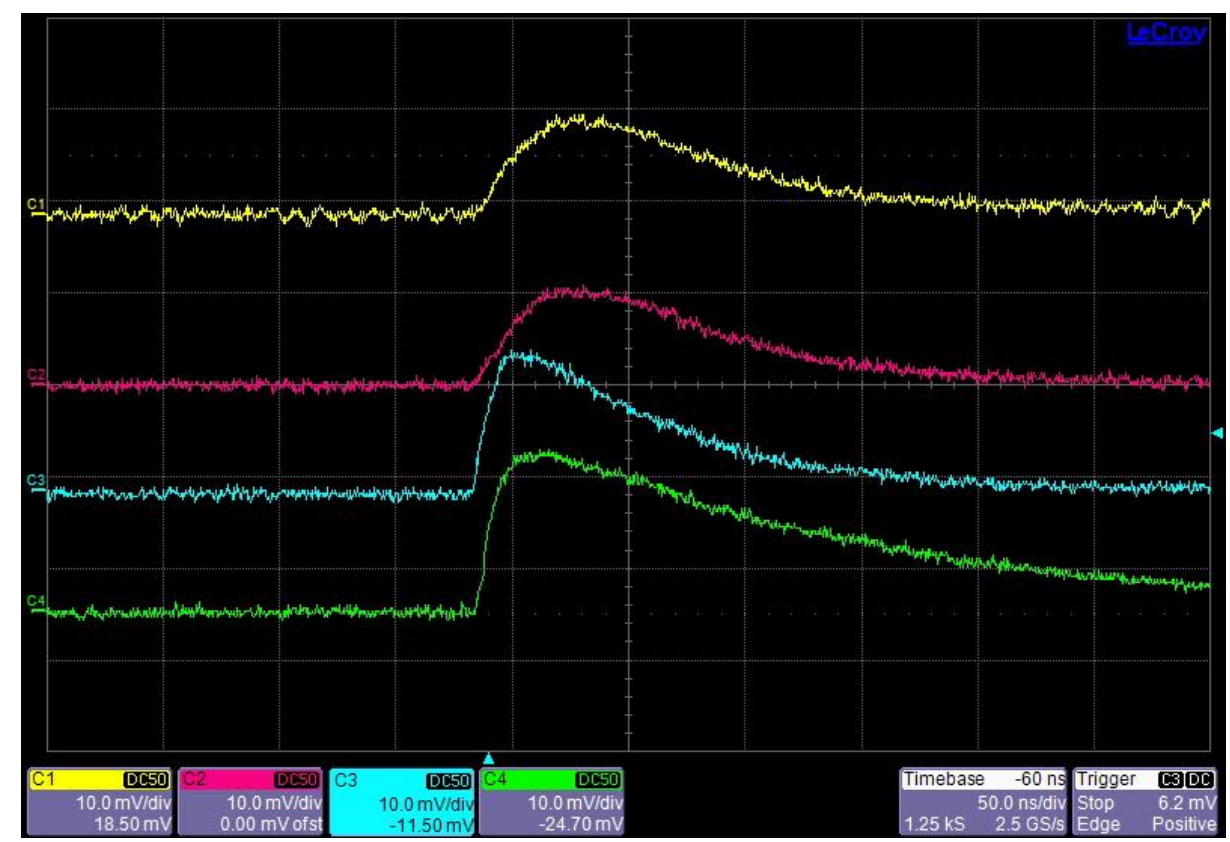

Figure 3: Signal display on a Lecroy $1 \mathrm{GHz}$ Wavesurfer MX104B oscilloscope for Hamamatsu SiPMT readout (bottom) and SenSL ones (top). Signals are from both edges of the scintillation counter under test.

violet laser diode, driven by an Avtech fast pulser ${ }^{4}$, is sent to the scintillation counter injection system (made of a total reflection prism) via a multimode fiber ${ }^{5}$. The laser spot is focused into the optical fiber (aligned by a micrometric x-y-z flexure system ${ }^{6}$ ) by a 10x Newport microscope objective. Light signals of different intensities were obtained both by tuning the laser parameters (e.g. amplitude and pulse width) and by changing the beam focussing onto the injection system. The laser beam is splitted by a broadband beamsplitter (BS) to give $50 \%$ of light on the fiber injection system and $50 \%$ on a monitoring detector. A fast Thorlabs DET10A/M photodiode (risetime $\sim 1$ ns) is used to monitor the laser intensity and give a reference start signal $\left(t_{0}\right)$ for the TDC system. The reference photodiode signal is amplified by a wideband CAEN A1433 inverting amplifier ${ }^{7}$. Four timing signals $\left(t_{0}, t_{s}, t_{1}, t_{2}\right)$ are then recorded by the acquisition system. $t_{1}, t_{2}$ refer to the two photodetectors at the edge of the scintillation counter, while $t_{0}$ refers to the reference photodiode

\footnotetext{
${ }^{4}$ Avtech AVO-9A-C laser diode driver, with $\sim 200$ ps risetime and a AVX-S1 output module. This system gave laser pulses at $\sim 408 \mathrm{~nm}$, with a pulse length between $\sim 400 \mathrm{ps}$ and $\sim 3 \mathrm{~ns}$ (as measured with a $6 \mathrm{GHz} 6604 \mathrm{~B}$ TEK scope) and repetition rate up to $1 \mathrm{MHz}$

${ }^{5}$ CERAM OPTEC UV 100/125 optical fiber, with a measured dispersion of $\leq 15 \mathrm{ps} / \mathrm{m}$, see [8]

${ }^{6}$ Thorlabs MBT613/M with a $4 \mathrm{~mm}$ escursion and $\sim 0.5 \mu \mathrm{m}$ resolution

${ }^{7}$ with $\sim 1.5 \mathrm{GHz}$ bandwidth and variable gain between $15 \mathrm{~dB}$ and $50 \mathrm{~dB}$
} 


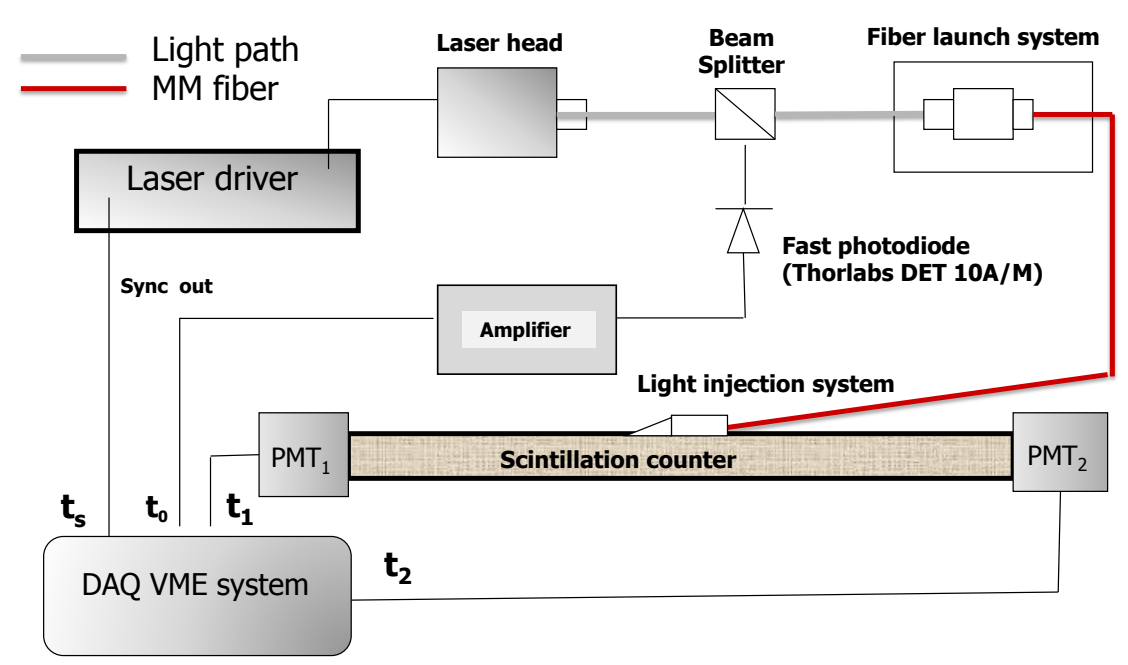

Figure 4: Laboratory setup used in the photomultipliers and SiPMT arrays readout characterization.

output and $t_{s}$ to the laser driver sync-out.

The acquisition system, in VME standard, is based on a VME-PCI interface card (CAEN V2718) and data written as binary files are then analyzed via the ROOT package [9]. The two signals: $t_{1}, t_{2}$ from the edges of the scintillation counter under test, are splitted $(50 \%-50 \%)$ by SUHNER passive splitters. They are sent to both a QADC line (CAEN V792) and a TDC line (with a CAEN V1290 TDC module after a CAEN V895 leading edge discriminator). Care has been taken to have comparable signals at both $\mathrm{L} / \mathrm{R}$ photodetectors, to avoid sizeable time-walk corrections.

The signal from the laser system was tuned in amplitude and pulse length to obtain a timing resolution for a counter equipped with two Hamamatsu R4998 PMTs, equivalent to the one obtained in a previous BTF testbeam at LNF, Frascati, for the same type of scintillation counters $[1]$.

From the gaussian fit of the $\Delta t_{-}$distribution ${ }^{8}$ of figure 5 a resolution of $43.2 \pm 0.4 \mathrm{ps}$ is obtained, to be compared with a BTF testbeam value of $\sim 45 \mathrm{ps}{ }^{9}$. This result established the conditions for the use of the laser system in the following tests. In figure 6 the intrinsic counter resolution $\sigma_{t}=\sigma_{\Delta t^{-}} / 2$ is reported as a function of the PMTs operating voltage $V_{o p}=V_{0}+\Delta V$. In normal operating conditions resolutions $\sigma_{t} \sim 40 \mathrm{ps}$ are obtained.

With the same tuning of the laser beam, data were taken with the SiPMT arrays in place of the R4998 photomultipliers. A scan varying the operating voltage $V_{o p}=V_{b r k}+$ overvoltage was

\footnotetext{
${ }^{8} \Delta t_{-}$is defined as the difference of the discriminated time signal from the two counter edges. The intrinsic TOF detector resolution may be then computed as $\sigma_{t}=\sigma_{\Delta t_{-}} / 2$

${ }^{9}$ with data taken with impinging electrons giving a 2-3 MIP signal
} 


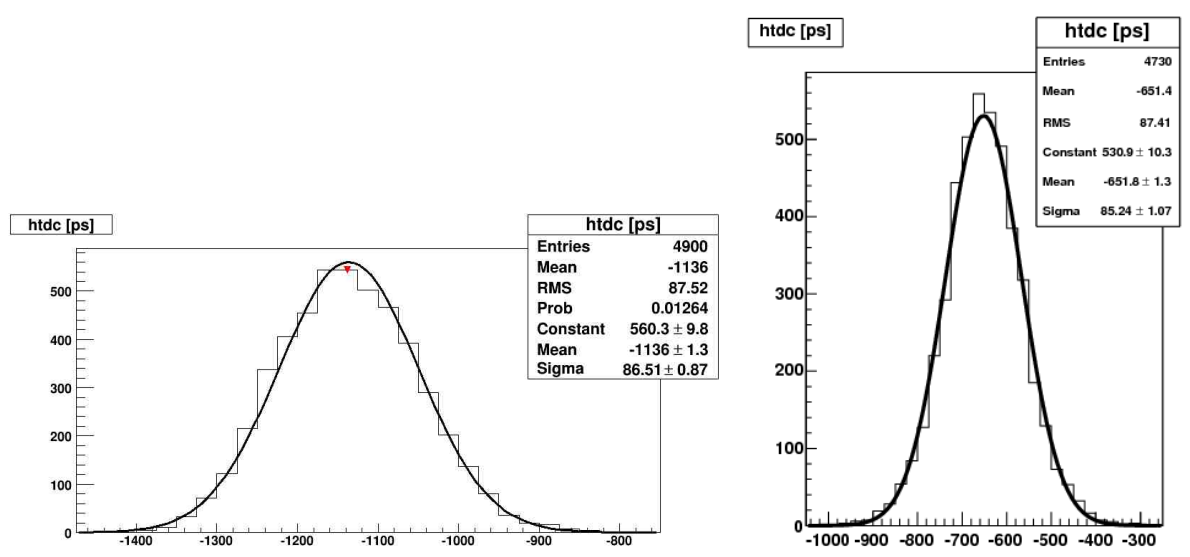

Figure 5: Left panel: distribution of $\Delta t_{-}$for the counter under test, equipped with R4998 PMTs at the two extremes. A gaussian fit is superimposed. Standard HV settings are used for both PMTs (-1750 V, -2250 V). Right panel: same distribution for the counter under test, equipped instead with Hamamatsu S11823344arrays. Operating voltage is $72.5 \mathrm{~V}$.

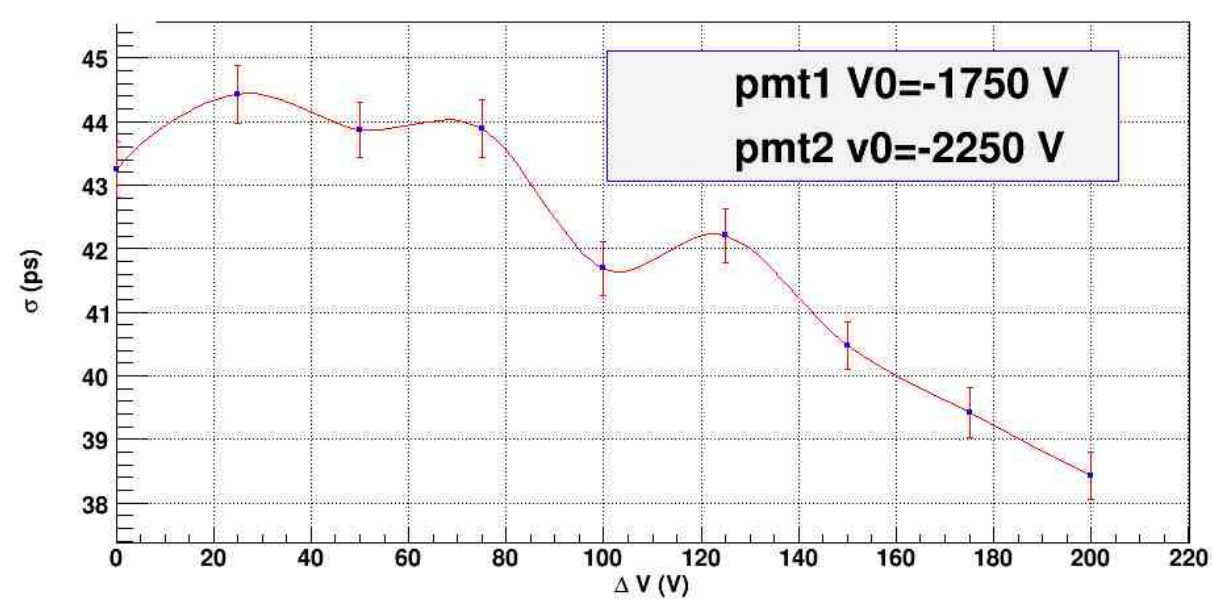

Figure 6: Intrinsic time resolution $\sigma_{t}$ for the counter under test equipped with conventional R4998 PMTs

done in both cases using a suitable threshold for the leading edge discriminator. The optimal value of this threshold $(\sim-80 \mathrm{mV})$ was established for both tested SiPMT arrays with scans at fixed operating voltages (see figure 7 ).

The best results are obtained with Hamamatsu arrays that are NUV extended, while the Advansid ones are RGB (with peak response at $\sim 500 \mathrm{~nm}$ ). Results are shown in figure 8 . For Hamamatsu S11828 arrays an intrinsic counter resolution $\sigma_{t} \sim 40 \mathrm{ps}$ is reached on an extended region of operating voltages, while for Advansid ASD-SiPM3S-4x4A arrays resolutions are at best $\sim 60$ ps.

As a limiting factor for the readout electronics of the used SiPMT arrays may be the bandwidth of the used home-made inverting amplifiers $(\sim 600 \mathrm{MHz})$, tests with a commercial wideband NIM 

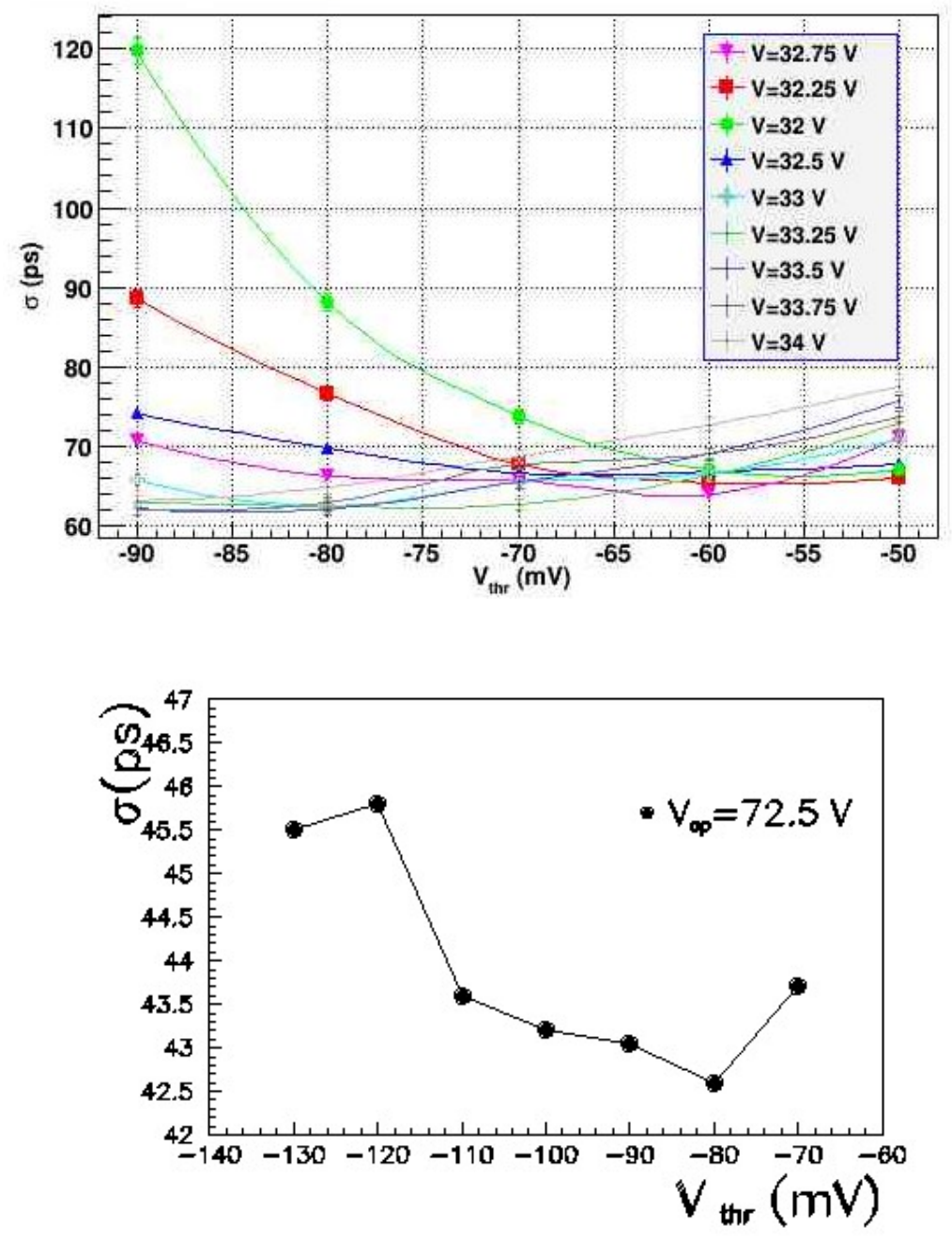

Figure 7: Top panel: intrinsic time resolution $\sigma_{t}$ at fixed voltages $V_{o p}$ obtained for the scintillation counter under test, equipped with Advansid arrays. as a function of the discriminator threshold. Bottom panel: same with Hamamatsu arrays readout at $V_{o p}=72.5 \mathrm{~V}$.

amplifier: a Phillips Scientific 774 module ${ }^{10}$ with external circuits for pole-zero compensation ${ }^{11}$ were done. Results are shown in figure 9 for the Hamamatsu SiPMT arrays. No appreciable improvements are seen.

\section{Conclusions}

Available SiPMT arrays are suitable as readout devices for time-of-flight detectors based on

\footnotetext{
${ }^{10}$ this module has gain of 50-100X, a bandwidth of $\sim 1.35 \mathrm{MHz}$ (with inverting Pulse Transformer Phillips Scientific 460)

${ }^{11}$ the nominal $50 \mathrm{X}$ gain was thus reduced to about $13 \mathrm{X}$
} 

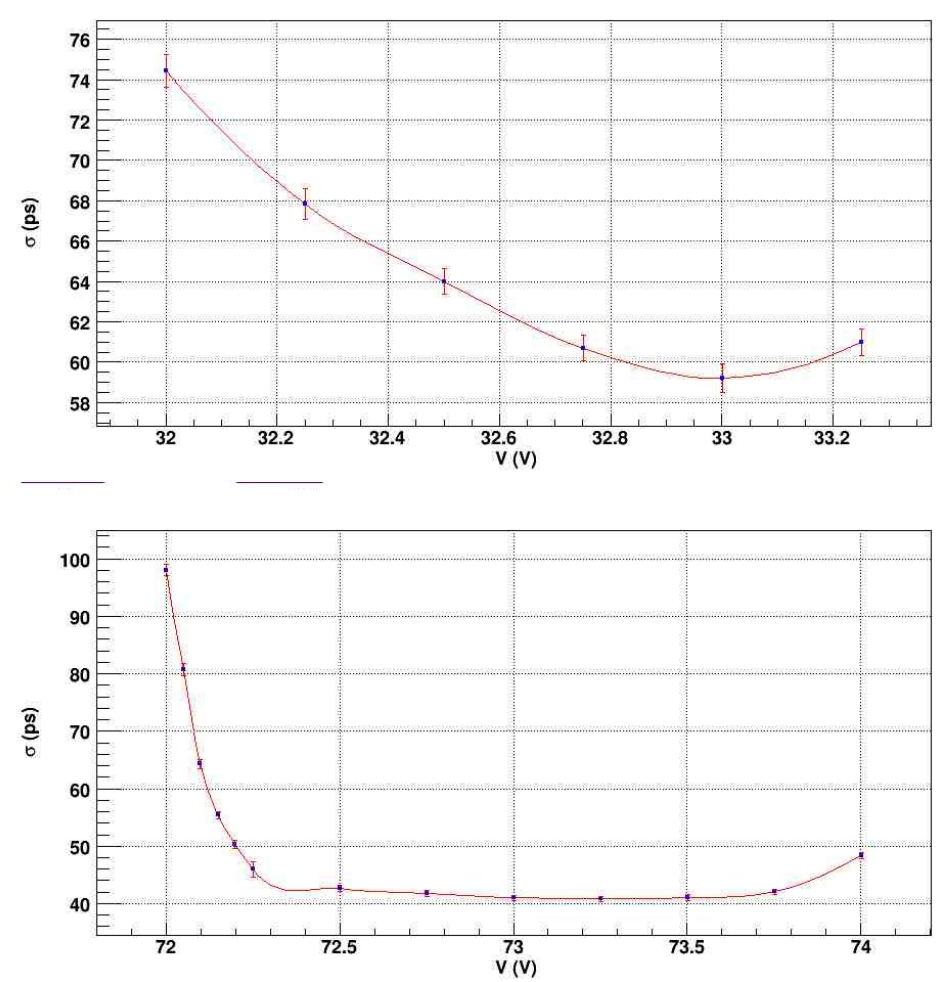

Figure 8: Intrinsic time resolution $\sigma_{t}$ vs the operating voltage $V_{o p}$ obtained for the scintillation counter under test, equipped with Hamamatsu S11828 (bottom panel) or Advansid ASD-SiPM3S-4x4A (top panel) SiPMT arrays.

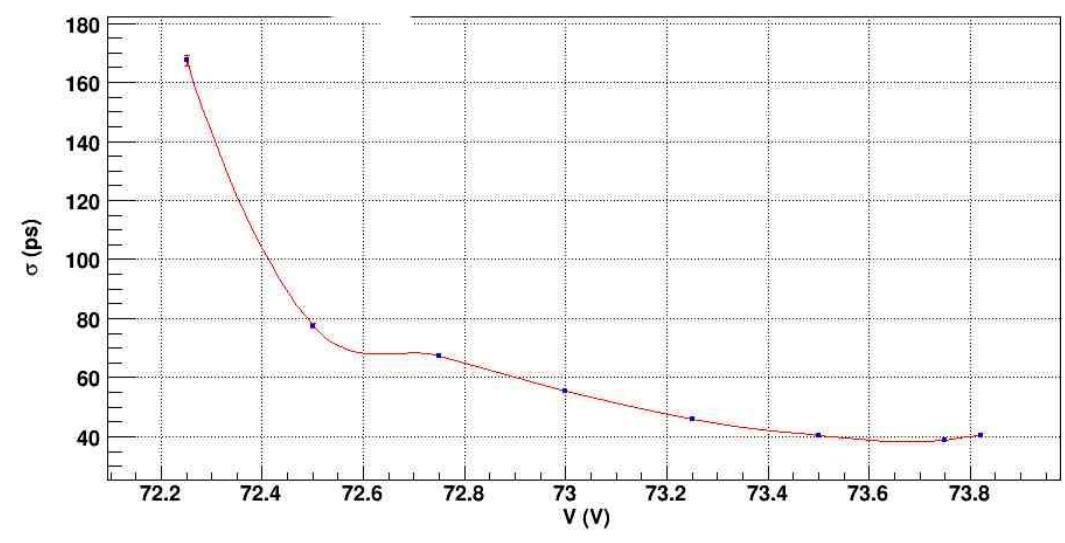

Figure 9: Intrinsic time resolution $\sigma_{t}$ vs the operating voltage $V_{o p}$ obtained for the scintillation counter under test, equipped with Hamamatsu S11828 SiPMT arrays. A PLS774 amplifier is used. 
scintillation counters. Timing resolutions $\sigma_{t}$, comparable with the best values obtained with photomultipliers, may be obtained by using a proper operating voltage. NUV extended Hamamatsu SiPMT arrays show better performances, as compared to RGB types as Advansid ASD-SiPM3S$4 \times 4 \mathrm{~A}$, for conventional scintillator readout.

\section{Acknowledgments}

We would like to acknowledge the skilfull work of Mr. R. Mazza, F. Chignoli INFN Milano Bicocca and Mr. D. Calabró, A. Freddi, C. Scagliotti, F. Vercellati INFN Pavia for the realization of the test setup and the relative mechanics and electronics. We would like to thanks Dr. N. Serra (Advansid), C. Piemonte (FBK/IRST) and M. Bombonati (Hamamatsu) for fruitful discussions and technical advice. We acknowledge also the essential work of our students L. Maver and G. Stringhini who took part with dedication and enthusiasm in the reported measurements.

\section{References}

[1] R. Bertoni et al., The design and commissioning of the MICE upstream time-of-flight system, Nucl. Instr. and Meth. A615 (2010) 14.

[2] M. Bonesini et al., Behaviour in Magnetic Fields of Fast Conventional and Fine-Mesh Photomultipliers, Nucl. Instr. and Meth. $\mathbf{A 6 9 3}$ (2012) 130.

[3] A. De Gerone et al., The MEG timing counter calibration and performance, Nucl. Instr. Meth. A 638 (2011)41

[4] S. Dussoni et al., The Timing Counter of the MEG experiment: Design and commissioning, Nucl. Instr. Meth. A617 (2010) 387

[5] M. Bonesini et al., Behaviour in high magnetic fields of fine-mesh photodetectors for fast time-of-flight detectors, Nucl. Instr. Meth. A567 (2006) 200

[6] M. Baldo-Ceolin et al., The time-of-fight TOFW detector of the HARP experiment: construction and performance, Nucl. Instr. Meth. A532 (2004) 548

[7] R. Bertoni et al., The construction of the TOF2 detector, MICE-NOTE-DET-0286, 2010, http://mice.iit.edu/micenotes/public/pdf/MICE0286/MICE0286.pdf M. Bogomilov et al. [MICE Coll], The MICE Muon Beam on ISIS and the beam-line instrumentation of the Muon Ionization Cooling Experiment, JINST 7(2012) P05009.

[8] M. Bonesini et al., Laser-based Calibration for the HARP Time of Flight System, IEEE Trans. Nucl. Sc. NS-50 (4) (2003) 1053.

[9] http://root.cern.ch 